\title{
Knockout of a-calcitonin gene-related peptide attenuates cholestatic liver injury by differentially regulating cellular senescence of hepatic stellate cells and cholangiocytes
}

\author{
Ying Wan ${ }^{1} \cdot$ Ludovica Ceci $^{2} \cdot$ Nan $_{\mathrm{Wu}^{2}} \cdot{\text { Tianhao } \mathrm{Zhou}^{2} \cdot \text { Lixian } \mathrm{Chen}^{2} \cdot \text { Julie Venter }^{2} \cdot \text { Heather Francis }}^{2,3,4}$.

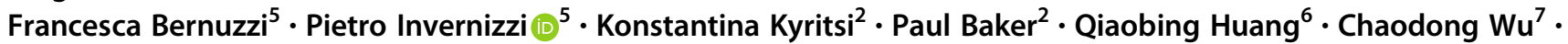 \\ Amelia Sybenga $^{8} \cdot$ Gianfranco Alpini $^{2,3,4} \cdot$ Fanyin Meng ${ }^{2,3,4,9} \cdot$ Shannon Glaser ${ }^{2,4}$
}

Received: 8 August 2018 / Revised: 22 November 2018 / Accepted: 6 December 2018 / Published online: 30 January 2019

(c) United States \& Canadian Academy of Pathology 2019

\begin{abstract}
$\alpha$-Calcitonin gene-related peptide ( $\alpha$-CGRP) is a 37-amino acid neuropeptide involved in several pathophysiological processes. $\alpha$-CGRP is involved in the regulation of cholangiocyte proliferation during cholestasis. In this study, we aimed to evaluate if $\alpha$-CGRP regulates bile duct ligation (BDL)-induced liver fibrosis by using a $\alpha$-CGRP knockout $\left(\alpha-\mathrm{CGRP}^{-/-}\right)$mouse model. $\alpha$-CGRP ${ }^{-/}$and wild-type (WT) mice were subjected to sham surgery or BDL for 7 days. Then, liver fibrosis and cellular senescence as well as the expression of kinase such as p38 and C-Jun N-terminal protein kinase (JNK) in mitogen-activated protein kinases (MAPK) signaling pathway were evaluated in total liver, together with measurement of cellular senescence in cholangiocytes or hepatic stellate cells (HSCs). There was enhanced hepatic expression of Calca (coding $\alpha$-CGRP) and the CGRP receptor components (CRLR, RAMP-1 and RCP) in BDL and in both WT $\alpha$-CGRP ${ }^{-/}$and BDL $\alpha-\mathrm{CGRP}^{-/-}$mice, $^{-}$ respectively. Moreover, there was increased CGRP serum levels and hepatic mRNA expression of CALCA and CGRP receptor components in late-stage PSC samples compared to healthy control samples. Depletion of $\alpha$-CGRP reduced liver injury and fibrosis in BDL mice that was associated with enhanced cellular senescence of hepatic stellate cells and reduced senescence of cholangiocytes as well as decreased activation of p38 and JNK MAPK signaling pathway. Cholangiocyte supernatant from BDL $\alpha-\mathrm{CGRP}^{-/-}$mice inhibited the activation and increased cellular senescence of cultured human HSCs (HHSCs) compared to HHSCs stimulated with BDL cholangiocyte supernatant. Taken together, endogenous $\alpha$-CGRP promoted BDL-induced cholestatic liver fibrosis through differential changes in senescence of HSCs and cholangiocytes and activation of p38 and JNK signaling. Modulation of $\alpha$-CGRP/CGRP receptor signaling may be key for the management of biliary senescence and liver fibrosis in cholangiopathies.
\end{abstract}

Ying Wan and Ludovica Ceci share the first authorship.

Drs. Gianfranco Alpini, Fanyin Meng, and Shannon Glaser share the senior authorship.

Present address for Drs. Bernuzzi and Invernizzi: Program for Autoimmune Liver Diseases, International Center for Digestive Health, Department of Medicine and Surgery, University of Milan-Bicocca, Milano, Italy.

Supplementary information The online version of this article (https:// doi.org/10.1038/s41374-018-0178-5) contains supplementary material, which is available to authorized users.

Gianfranco Alpini

gialpini@iu.edu

gianfranco.alpini@va.gov

Extended author information available on the last page of the article

\section{Introduction}

Liver fibrosis is a common consequence of acute damage and chronic reversible wound-healing response to liver injury [1]. Progressive liver fibrosis results in cirrhosis $[2,3]$, which is a major risk factor for hepatocellular carcinoma (HCC) [4]. However, the mechanisms of liver fibrosis (a major health problem worldwide) are not completely elucidated. A hallmark of liver fibrosis is the activation and transdifferentiation of hepatic stellate cells (HSCs), which also serve as major producer of fibrogenic extracellular matrix (ECM) [5]. Therefore, targeting the activation of HSCs may be a promising approach to reduce liver fibrosis and the consequent risk of liver cirrhosis.

Cellular senescence is a stable form of cell cycle arrest program, which may limit the proliferative activity of 
cells. Recent studies have demonstrated that senescent cells exhibit large and flattened morphology, and accumulate senescence-associated $\beta$-galactosidase (SA- $\beta$-gal) activity [6]. Some studies have shown that the senescence of activated HSCs down-regulates extracellular matrix synthesis to limit liver fibrosis [6, 7]. Cholangiocytes are the target cells of cholangiopathies including primary sclerosing cholangitis (PSC) and primary biliary cholangitis (PBC) [8] and in animal models of cholestasis including bile duct ligation (BDL) and the multidrug resistance protein $2\left(\mathrm{Mdr}^{--}\right)$mouse model of PSC $[9,10]$. Senescence of cholangiocytes has been shown to be an important player in the development of cholestatic liver injury and biliary fibrosis $[11,12]$. During tissue injury, neuropeptides such as tachykinins and $\alpha$-calcitonin generelated peptide ( $\alpha$-CGRP) are released by tissue peripheral nerve terminals and have local functions such as neurogenic inflammation [13]. $\alpha$-CGRP is a 37 -amino acid peptide that is primarily localized to sensory neurons. $\alpha$-CGRP is also distributed in various tissues and CGRP receptors are found in the brain, heart, liver, lung and spleen [14, 15]. CGRP receptor is composed of three subunits: calcitonin receptor-like receptor (CRLR), receptor activity-modifying protein 1 (RAMP-1) and receptor component protein (RCP). CGRP levels are higher in the serum of cirrhotic patients compared to healthy controls [16]. Studies have shown that: (i) $\alpha$-CGRP stimulate cholangiocyte proliferation during BDL-induced cholestasis; [15] and (ii) CGRP contributes to the activation of protein kinase $\mathrm{C}$ (PKC) resulting in activation of the mitogen-activated protein kinases (MAPKs) such as extracellular signal-regulated kinase (ERK), C-Jun N-terminal protein kinase (JNK) and p38 kinase and it is involved in the stimulation of biliary hyperplasia as well as the development of renal fibrosis $[17,18]$. On the basis of these findings, we performed studies aimed to evaluate the intracellular mechanisms by which $\alpha$-CGRP modulates biliary damage and liver fibrosis during cholestasis.

\section{Methods and materials}

\section{Materials}

Reagents were purchased from Sigma-Aldrich Chemical Co. (St. Louis, MO) unless otherwise indicated. The mouse monoclonal antibody against receptor activitymodifying protein 1 (RAMP-1) was purchased from Santa Cruz Biotechnology (Dallas, TX). The following antibodies were purchased from Abcam (Burlingame, CA): (i) rabbit polyclonal antibodies against cyclin-dependent kinase inhibitor 2A (p16) (Ab189034) and $\alpha$-smooth muscle actin ( $\alpha$-SMA) (Ab5694); (ii) rabbit monoclonal antibody against desmin (marker of HSCs) [10, 19, 20] (Ab185033); and (iii) rabbit polyclonal antibody to fibronectin (Ab2413). The rat monoclonal antibody against cytokeratin-19 (CK-19) was purchased from TROMA-III. The mouse antibody against p16 (sc-166760) was purchased from Santa Cruz Biotechnology. The antibodies against SAPK/JNK (9252), p-JNK (4668), p38 MAPK (9212 S) and p-p38 MAPK (9211) were purchased from Cell Signaling Technology (Danvers, MA). CGRP EIA kits (for detecting CGRP levels) were purchased from Peninsula Laboratories International, Inc. (San Carlos, CA). Rat $\alpha$-CGRP was purchased from Phoenix Pharmaceuticals, Inc. (Burlingame, CA). The CGRP receptor antagonist $\left(\mathrm{CGRP}_{8-37}\right)$ [15] was purchased from Peninsula Laboratories International, Inc. (San Carlos, CA). The RNeasy Mini Kit for RNA purification and all selected primers were purchased from Qiagen (Valencia, CA). The iScript cDNA Synthesis Kit (170-8891) and iTaq Universal SYBR Green Supermix (172-5124) were purchased from Bio-Rad (Hercules, CA). All primers for quantitative polymerase chain reaction (qPCR) were purchased from Qiagen (Valencia, CA); specific information about the primers used in this project is listed in Supplemental Table 1.

\section{Animal models}

All animal experiments were performed in accordance with protocols approved by the Baylor Scott \& White Healthcare IACUC Committee. C57BL/6 wild-type (WT) mice were purchased from Charles River (Wilmington, MA). The $\alpha$-CGRP knockout $\left(\alpha-\mathrm{CGRP}^{--}\right)$mouse model was generated as described [21]. The mice were maintained in a temperature-controlled environment $\left(20-22{ }^{\circ} \mathrm{C}\right)$ with 12:12-h light/dark cycles and fed with standard mouse chow with free access to drinking water ad libitum. Male WT and $\alpha$-CGRP ${ }^{-/-}$mice (approximately 25-30 g, 10-12 weeks of age) underwent BDL [22] or sham surgery for 1 week before collecting serum, total liver samples, cholangiocytes and cholangiocyte supernatant.

\section{Human healthy control and PSC samples}

The serum $(n=3)$ and liver tissue $(n=4)$ samples from healthy human and patients with early $(n=2)$ and advanced stage (3-4, $n=3$ ) of PSC (Table 1) were obtained from Dr. Pietro Invernizzi under a protocol approved by the Ethics Committee of the Humanitas Research Hospital; which was reviewed by the Veterans' Administration IRB and International Research Committee. The use of human tissue was approved by the Texas A\&M HSC College of Medicine Institutional Review Board. 


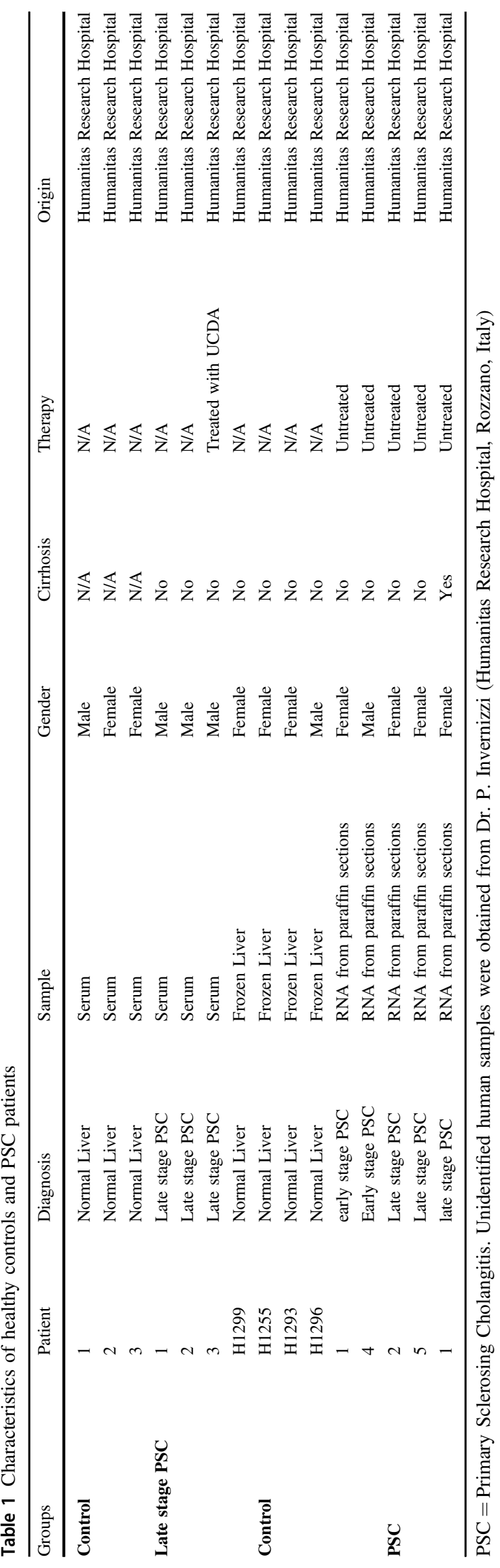
Isolated mouse cholangiocytes and HSCs, and cell
lines of immortalized murine cholangiocytes and human hepatic stellate cells

Mouse cholangiocytes were obtained by immunoaffinity separation [22, 23]. Mouse HSCs were isolated by laser capture microdissection (LCM) using an antibody against desmin, a marker of HSCs [24]. The RNA from LCMisolated HSCs was extracted with the Arcturus PicoPure RNA isolation kit (Thermo Fisher Scientific CO, Mountain View, CA) according to the instructions provided by the vendor. Expression of fibrosis and senescence markers was measured in these cells by quantitative polymerase chain reaction (qPCR).

The in vitro studies were performed in human hepatic stellate cell lines (HHSCs) and murine immortalized biliary cell lines (IMCLs) [23] that were purchased from ScienCell Research Laboratories (Carlsbad, CA). These cells were seeded into six-well plates and treated with $0.2 \%$ BSA (basal) or $\alpha$-CGRP $\left(10^{-9} \mathrm{M}\right)$ for $24 \mathrm{~h}$ in the absence/presence of $\mathrm{CGRP}_{8-37}(1 \mu \mathrm{M})$ [15]. Cells were harvested and the expression of fibrosis and senescence genes were evaluated by qPCR. In other in vitro experiments, HHSCs were treated with cholangiocyte supernatant from the aforementioned animal groups before evaluating the mRNA and protein expression of fibrosis and senescence markers by qPCR and by immunofluorescence. All of these studies were performed in triplicate. Data are expressed as relative mRNA levels \pm SEM of the selected gene to glyceraldehyde-3-phosphate dehydrogenase (GAPDH) ratio.

\section{Evaluation of CGRP serum levels in human PSC samples and hepatic mRNA expression of Calca and CGRP receptor components in cholestatic mouse models and PSC samples}

CGRP serum levels from human healthy controls and early and late-stage PSC patients $(n=5)$ were measured by EIA kits (see above). Total RNA was extracted paraffin-embedded section samples from five PSC patients by the kit RNeasy FFPE kit (Qiagen). The RNA from mouse liver was extracted by using the RNeasy Mini Kit for RNA purification (Qiagen). The mRNA expression of Calca (coding $\alpha$-CGRP), CGRP receptor components (CRLR, RAMP-1 and RCP) in isolated cholangiocytes of the selected mouse samples $(n=4)$ and in total liver of PSC $(n=5)$ and healthy control samples $(n=4)$ was evaluated by qPCR. Data are expressed as relative mRNA levels \pm SEM of the selected gene to GAPDH ratio. 

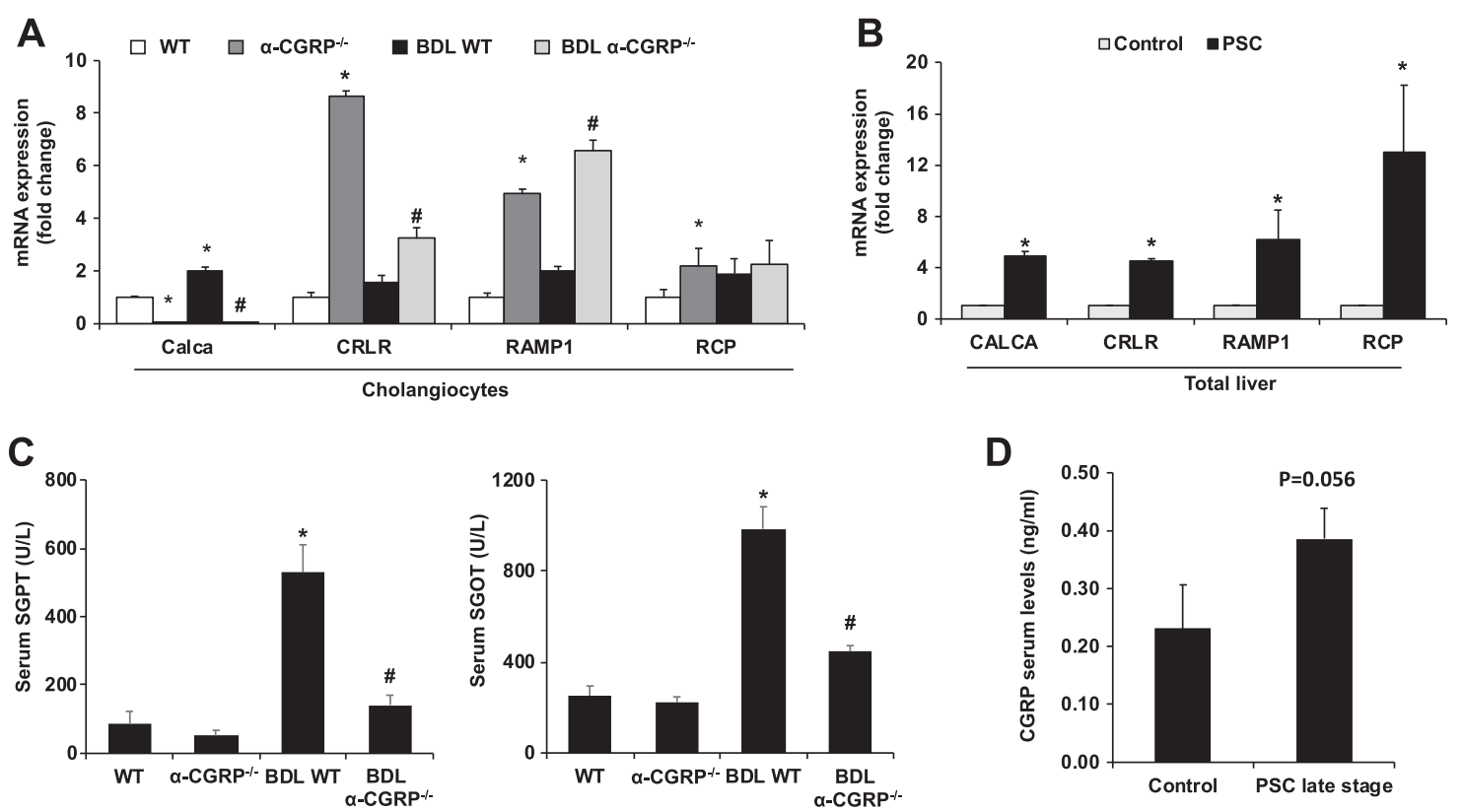

Fig. 1 Increased expression of $\alpha$-CGRP contributes to liver injury during cholestasis. A Lack of $\alpha$-CGRP induced CGRP receptor components expression (CRLR, RAMP-1, and RCP) in $\alpha-\mathrm{CGRP}^{-/-}$and $\mathrm{BDL} \alpha-\mathrm{CGRP}^{-/-}$mice compared to WT and BDL WT mice, respectively, in isolated cholangiocytes $(n=4)$. B Hepatic mRNA expression of CALCA $(n=1)$ and CRLR $(n=1), \operatorname{RAMP}-1(n=5)$, and $\operatorname{RCP}$

$(n=5)$ were increased in total liver from PSC patients compared to healthy controls $(n=4)$. C Knockout of $\alpha$-CGRP decreased SGPT and SGOT serum levels in BDL mice $(n=3)$. D CGRP serum levels of PSC patient. CGRP serum levels were higher in PSC $(n=5)$ patients than their control groups $(n=4) .{ }^{*} p<0.05$ vs. WT mice or health control samples; ${ }^{\#} p<0.05$ vs. BDL WT mice

\section{Assessment of serum chemistry and liver fibrosis}

The serum levels of alanine aminotransferase (SGTP), aspartate aminotransferase (SGOT) and total bilirubin were measured by a Catalyst One Chemistry Analyzer from IDEXX Laboratories, Inc. (Westbrook, ME). In addition, we measured the serum levels of IL-6 and TGF$\beta 1$ in cholangiocyte supernatant by the BD OptEiA ${ }^{\mathrm{TM}}$ Mouse IL-6 ELISA Kit and the TGF- $\beta 1$ ELISA kit (Affymetrix Inc., Santa Clara, CA), respectively [10]. Liver histology was evaluated in paraffin-embedded liver sections (4-5 $\mu \mathrm{m}$ thick) from the selected groups of mice by hematoxylin and eosin (H\&E) staining. Observations were processed by Image-Pro Plus software (Media Cybernetics) in a blinded fashion by a board-certified pathologist. Liver fibrosis was measured by: (i) Sirius red staining (ten different fields analyzed from three different samples from three animals) and Masson trichrome staining in liver sections ( $4-5 \mu \mathrm{m}$ thick); (ii) Hydroxyproline levels in total liver samples using the Hydroxyproline Assay Kit (MAK008; Sigma-Aldrich); and (iii) immunoblots for $\alpha$-SMA in total liver. We also evaluated the mRNA expression of: (i) TGF- $\beta 1$ and Smad2 in total liver; (ii) $\alpha$-SMA, Fn1, and Col1 $\alpha 1$ in total liver and isolated cholangiocytes; and (iii) Col1 $\alpha 1$ in LCM-isolated HSC by qPCR (20).

\section{Measurement of cellular senescence}

Cellular senescence was measured in frozen liver sections $(10 \mu \mathrm{m}$ thick $)$ by staining for senescence-associated $\beta$-galactosidase (SA- $\beta$-gal) using a commercially available kit (MilliporeSigma, Billerica, MA); all the experiments were performed in three different liver samples from three different animals. Cellular senescence was also evaluated by measuring the mRNA expression of the senescent markers: (i) p16, p21, CCL2 and PAI-1 in total liver samples; (ii) p16 and p21 in isolated cholangiocytes and HSCs by qPCR [23]; and (iii) p16 in total liver by immunoblots. In addition, other senescent-associated secretory phenotypes (SASP) such as IL-6, IL-8, IL-1 $\beta$, and matrix metalloproteinase-2 (MMP2) was measured in isolated cholangiocytes. Immunoreactivity for p16 was also measured by immunofluorescence in frozen liver sections $(6-8 \mu \mathrm{m}$ thick); the images were analyzed by using Leica AF 6000 Modular Systems.

\section{Measurement of MAPK expression in total liver}

Protein was extracted from total liver with lysis buffer and quantified by the bicinchoninic acid method (Pierce Biotechnology, Inc., Rockford, IL). Then, the expression of p-JNK and p-p38 MAPK (expressed as ratio to total protein 

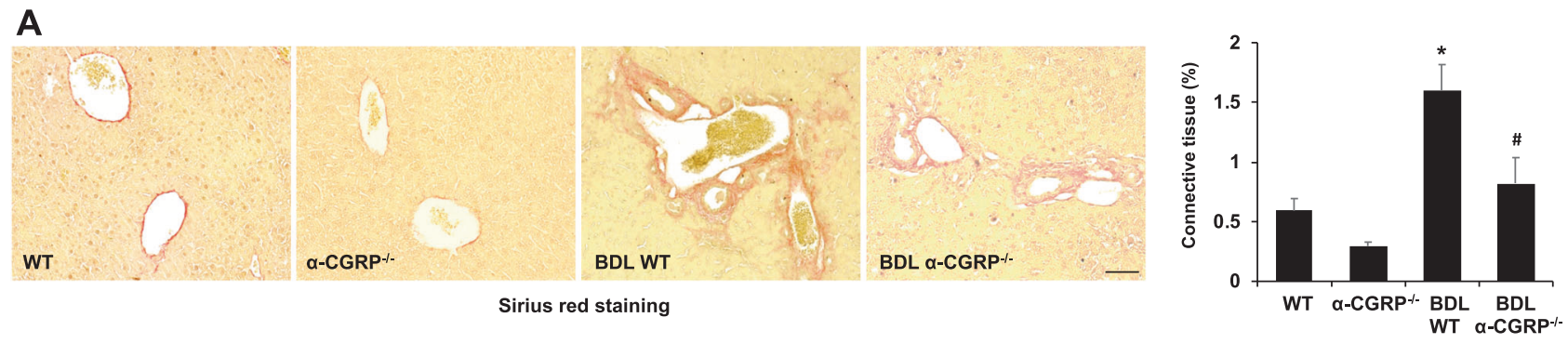

B

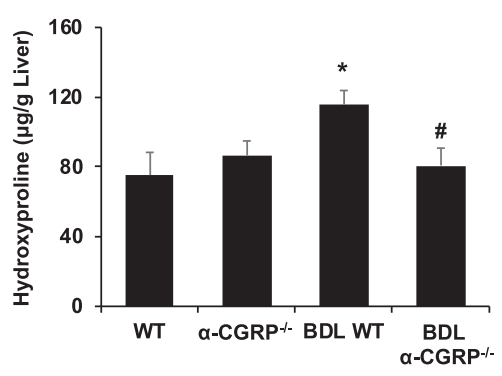

Fig. 2 Lack of $\alpha$-CGRP decreases cholestatic liver fibrosis induced by BDL. A There was enhanced liver fibrosis in BDL WT compared to WT mice, but reduced liver fibrosis in BDL $\alpha-\mathrm{CGRP}^{-/}$mice compared to BDL WT mice $(n=3$, Orig., magnification, $20 \times$; scale bar $=$ $50 \mu \mathrm{m})$. B Hydroxyproline levels in total liver samples from BDL

expression of JNK and p38) was evaluated by immunoblots [24] and visualized and quantified using the LI-COR Odyssey Infrared Imaging System (LI-COR Bioscience, Lincoln, NE).

\section{Statistical analysis}

All data are expressed as the mean \pm SEM. Differences between groups were analyzed by Student's unpaired $t$-test when two groups were analyzed or analysis of variance followed by Tukey post-hoc test for multiple comparisons when more than two groups were analyzed using SPSS software 22.0 (Armonk, NY). A value of $p<0.05$ was considered significant.

\section{Results}

\section{Enhanced hepatic mRNA expression of Calca, increased liver injury in BDL mice, and enhanced hepatic expression of CALCA and CGRP receptor components as well as CGRP serum levels in human PSC samples}

There was increased mRNA expression of Calca in isolated cholangiocytes from BDL WT compared to control WT mice, increase that was reduced in $\mathrm{BDL} \alpha-\mathrm{CGRP}^{-/}$mice compared to BDL WT mice (Fig. 1A). Furthermore, there was enhanced mRNA expression of the CGRP receptor components (CRLR, RAMP-1 and RCP) in cholangiocytes from $\alpha-\mathrm{CGRP}^{-/}$mice compared to WT mice (Fig. 1A). The expression of CRLR and RAMP-1 was also increased in cholangiocytes from BDL $\alpha-\mathrm{CGRP}^{-/-}$compared to BDL WT mice (Fig. 1A). All together, these data suggest that the lacking of $\alpha$-CGRP expression in BDL mice induces a compensatory response from cholangiocytes by increasing the expression of CGRP receptor components. The mRNA expression of CALCA and CGRP receptor components increased in total liver samples from PSC patients compared to healthy controls (Fig. 1B). The serum levels of SGPT and SGOT (Fig. 1C) and total bilirubin (Supplemental Fig. 2A) were also increased in BDL WT mice compared to WT mice, but significantly decreased in $\mathrm{BDL} \alpha-\mathrm{CGRP}^{-/-}$mice compared to BDL WT mice. Furthermore, the CGRP serum levels were higher in PSC patients compared to healthy controls (Fig. $1 \mathrm{D}$ ). There were no differences in the serum levels of IL-6 between normal WT and $\mathrm{CGRP}^{-/-}$mice, but there was a significant reduction of IL-6 serum levels in $\mathrm{BDL}_{\mathrm{CGRP}}{ }^{-/}$mice compared to BDL WT mice (Supplemental Fig. 2B).

\section{Lack of a-CGRP reduces liver fibrosis and activation of MAPK signaling pathway during BDL-induced cholestasis}

Previously, we have demonstrated reduced intrahepatic biliary mass in BDL $\alpha-\mathrm{CGRP}^{-/-}$mice compared to BDL WT 


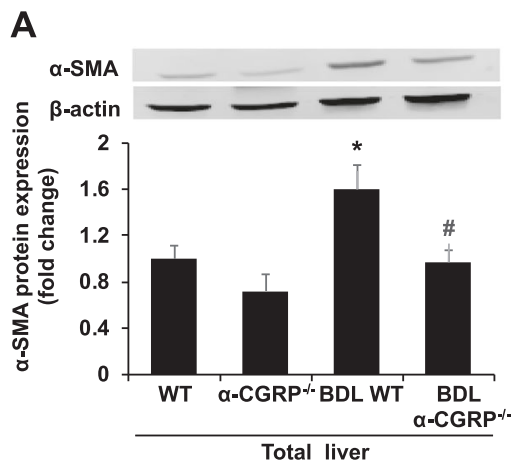

B
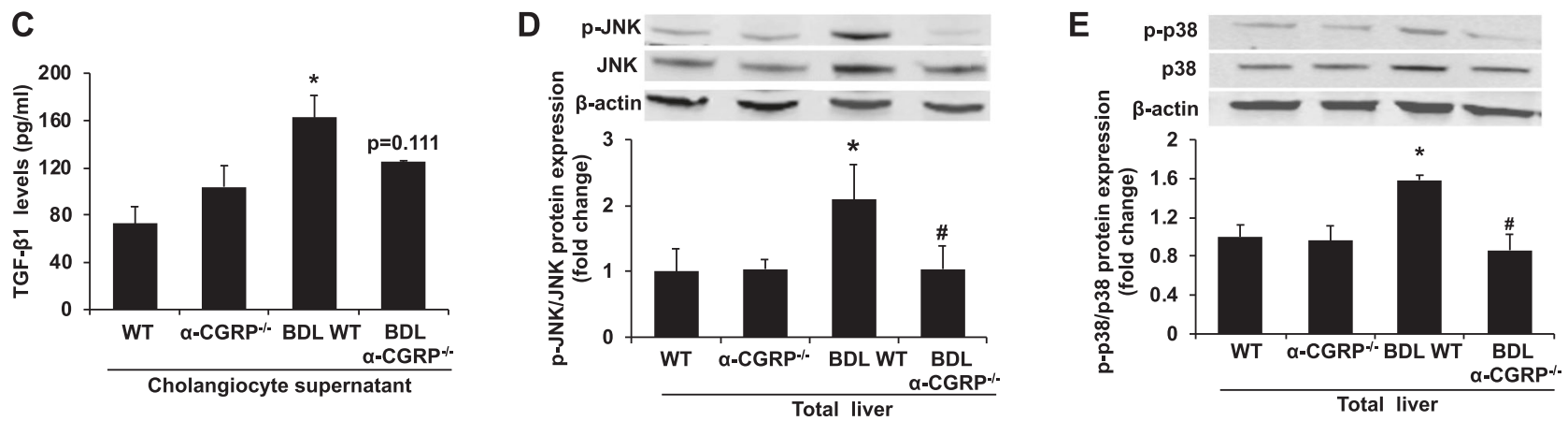

Fig. 3 Lack of $\alpha$-CGRP reduces BDL-induced fibrosis marker expression. A There was decreased protein expression of $\alpha$-SMA in total liver from $\mathrm{BDL} \alpha-\mathrm{CGRP}^{-/-}$mice compared to BDL WT mice $(n=3)$. B There was increased mRNA expression of TGF- $\beta 1$ and Smad2 in total liver from BDL WT compared to WT mice, whereas the expression of these two genes was reduced in $\mathrm{BDL} \alpha-\mathrm{CGRP}^{-/-}$mice compared to BDL WT mice $(n=3)$. C TGF- $\beta 1$ levels were higher in cholangiocyte supernatant from BDL WT mice than those in normal cholangiocyte supernatant from WT mice but decreased in cholangiocyte supernatant from BDL $\alpha-\mathrm{CGRP}^{-/-}$mice compared to BDL WT mice. D, E The protein expression of $\mathbf{D}$ p-JNK and $\mathbf{E}$ p-p38 in total liver was increased in BDL WT mice compared to WT mice, whereas decreased in BDL $\alpha-\mathrm{CGRP}^{-/-}$mice compared to BDL WT mice $(n=3)$. ${ }^{*} p<0.05$ vs. WT mice; ${ }^{*} p<0.05$ vs. BDL WT mice mice [15]. Thus, we hypothesize that $\alpha$-CGRP may be a profibrotic factor during BDL-induced liver fibrosis. By H\&E staining, severe histological changes were observed in liver sections of BDL mice compared to WT mice, which was reduced in BDL $\alpha-\mathrm{CGRP}^{-/}$mice (Supplemental Fig. 1A). While the hepatic architecture of normal WT mice was normal, we observed sinusoidal congestion and reactive nuclear changes in hepatocytes in the liver of normal $\alpha$ $\mathrm{CGRP}^{-/-}$mice; rare necrotic hepatocytes were present. No inflammation, ductular reaction or definite fibrosis was identified (Supplemental Fig. 1A). Liver sections of BDL WT mice show portal areas expanded by a mild ductular reaction, with a mild patchy periductal concentric fibrosis and acute inflammation extending into the bile duct epithelium. There was mild, patchy activity at the limiting plate with few small and rare large islands of necrotic hepatocytes in the lobules. There was patchy mild lobular acute inflammation and focal mild collapse. A portion of extrahepatic bile duct is present with marked granulation tissue and foreign body giant cell reaction (Supplemental Fig. 1A). Liver sections of liver of BDL $\alpha-\mathrm{CGRP}^{-/-}$mice show portal areas expanded by marked ductular reaction and periductal concentric fibrosis. There was mild acute inflammation extending into the bile duct epithelium with patchy activity at the limiting plate. There were large islands of necrotic hepatocytes in the lobules and single cell necrosis with early bridging fibrosis (Supplemental Fig. 1A).

By Sirius red staining, there was enhanced collagen hepatic deposition in BDL WT mice compared to WT mice, which was significantly reduced in BDL $\alpha-\mathrm{CGRP}^{-/-}$compared to BDL WT mice (Fig. 2A); similar results were obtained by Masson trichrome staining (Supplemental Fig. 1B). Moreover, by measurement of hydroxyproline levels in total liver samples there was reduced collagen deposition in BDL $\alpha-\mathrm{CGRP}^{-/-}$mice compared to BDL WT mice (Fig. 2B). By qPCR, there was enhanced expression of $\alpha$-SMA and fibronectin (Fn1) in total liver from BDL WT mice compared to WT mice, increase that was reduced in BDL $\alpha$-CGRP ${ }^{-/-}$mice compared to BDL WT mice (Fig. 2C, D). Furthermore, BDL-induced $\alpha$-SMA protein expression was decreased in total liver samples from BDL $\alpha$-CGRP ${ }^{-1-}$ mice compared to BDL WT mice (Fig. 3A). There was: (i) decreased mRNA expression of: TGF- $\beta 1$ and Smad 2 in total liver (Fig. 3B); and (ii) reduced TGF- $\beta 1$ levels in cholangiocyte supernatant (Fig. 3C) from BDL $\alpha$-CGRP ${ }^{-/-}$ mice compared to BDL WT mice. Additionally, the MAPK signaling pathway was activated during BDL, which was 

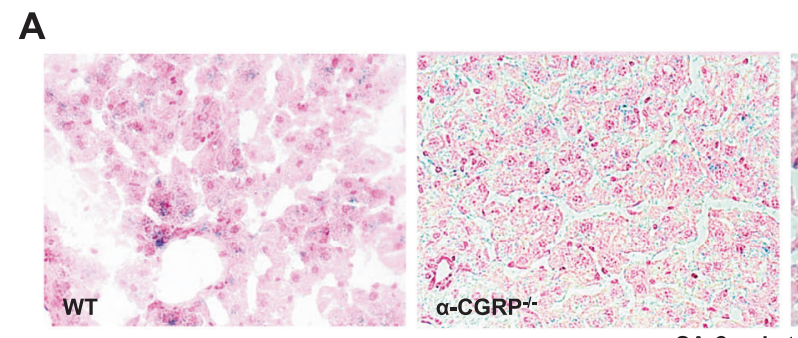

SA- $\beta$-gal staining

B

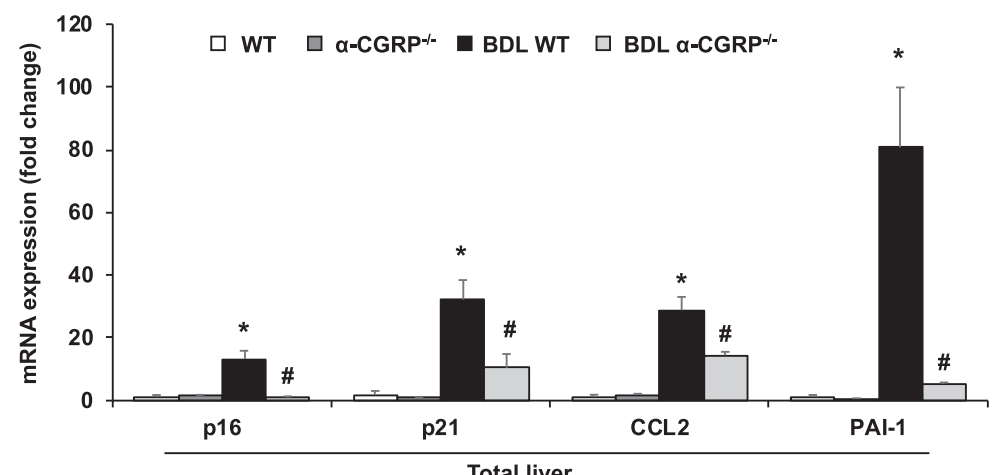

Total liver

Fig. 4 Lack of $\alpha$-CGRP reduces BDL-induced cellular senescence. A Cellular senescence was enhanced in BDL WT mice compared to WT mice but reduced in $\mathrm{BDL} \alpha-\mathrm{CGRP}^{-/-}$mouse liver compared to $\mathrm{BDL}$ WT mice, which was verified by SA- $\beta$-gal staining in liver sections ( $n=4$, Orig., magnification, $40 \times$; scale bar $=100 \mu \mathrm{m}) . \mathbf{B}, \mathbf{C}$ The

evidenced by enhanced expression of p-JNK and p-p38 MAPK in total liver of BDL WT mice compared to WT mice, expression that was partly reduced in BDL $\alpha-\mathrm{CGRP}^{-/-}$ mice (Fig. 3D, E).

\section{Lack of a-CGRP decreases BDL-induced cellular senescence in total liver and isolated cholangiocytes, but increases cellular senescence in HSCs from BDL mice}

Since biliary senescence contributes to liver fibrosis in PSC and PBC [8] as well as in cholestatic mouse models [10], we evaluated if the changes of cellular senescence underlie the effect of $\alpha$-CGRP on liver fibrosis during cholestasis. By SA- $\beta$-gal staining, we demonstrated that there was enhanced cellular senescence in liver sections from BDL WT mice compared to WT mice (Fig. 4A). On the contrary, cellular senescence was reduced in total liver from BDL $\alpha$-CGRP ${ }^{-/-}$mice compared to BDL WT mice (Fig. 4A). By qPCR, there was: (i) increased expression of p16, p21, CCL2, and PAI-1 in total liver from BDL WT mice compared to WT mice; and (ii) decreased expression of these senescent genes in total liver samples from BDL $\alpha$-CGRP ${ }^{-/}$ mice compared to BDL WT mice (Fig. 4B). By immunoblots, decreased expression of p16 was observed in total
mRNA expression of $\mathbf{B}$ p16, p21, and CCL2 and PAI-1 as well as $\mathbf{C}$ p16 protein levels were increased in total liver from BDL WT mice compared to WT mice but decreased in total liver from BDL $\alpha$-CGRP ${ }^{-/-}$mice compared to BDL WT mice $(n=3) . * p<0.05$ vs. WT mice; ${ }^{\#} p<0.05$ BDL WT mice

liver from $\mathrm{BDL} \alpha-\mathrm{CGRP}^{-/}$mice compared to $\mathrm{BDL}$ WT mice (Fig. 4C). Furthermore, the expression of fibrosis and senescence markers increased in cholangiocytes from BDL WT mice compared to normal mice; these changes were partially reduced in BDL $\alpha-\mathrm{CGRP}^{--}$mice (Fig. 5A, B). Moreover, reduced expression of IL-6, IL-8, IL-1 $\beta$, and MMP2 was observed in cholangiocytes from BDL $\alpha-\mathrm{CGRP}^{-/-}$mice compared to BDL WT mice (Fig. 5C). By immunofluorescence in liver sections, increased expression of p16 in cholangiocytes was observed from BDL WT mice compared to WT mice, however, the expression of p16 was decreased in cholangiocytes from BDL $\alpha$-CGRP ${ }^{-/-}$mice compared to BDL WT mice (Fig. 5D). Conversely, senescence gene (p16 and p21) and protein (p16) expression was increased in $\mathrm{HSCs}$ from BDL $\alpha-\mathrm{CGRP}^{-/-}$mice compared to BDL WT mice (Fig. 6B). It was observed decreased co-localization of the HSC specific marker, des$\min [20,25,26]$, and p16 in BDL mice compared to BDL $\alpha$-CGRP ${ }^{-/-}$mice, which suggests that senescence of HSCs is reduced in $\mathrm{BDL}$ mice compared to $\mathrm{BDL} \alpha-\mathrm{CGRP}^{-/-}$mice (Fig. 6C). Meanwhile, depletion of $\alpha$-CGRP decreased the expression of Col1 $\alpha 1$ in HSCs during BDL- induced liver injury (Fig. 6A). The fact there are no changes of fibrosis and senescence in $\alpha$-CGRP ${ }^{-/}$mice compared with WT mice (Figs. 4-5) is likely due to the fact that there is no activation 


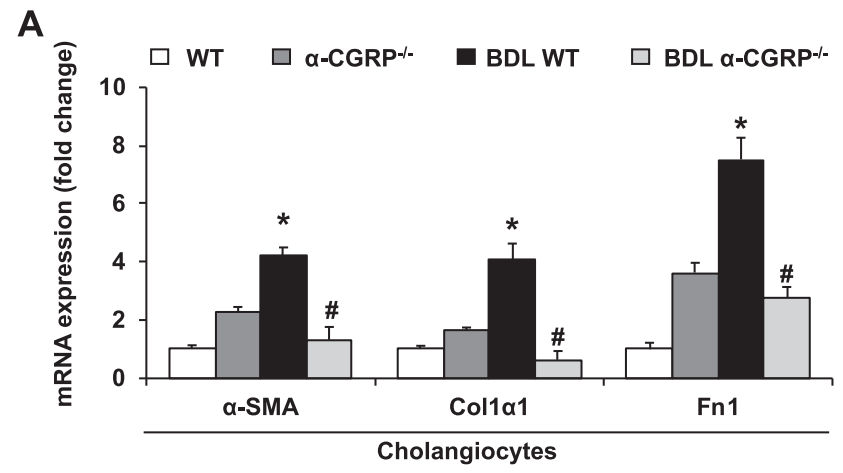

C

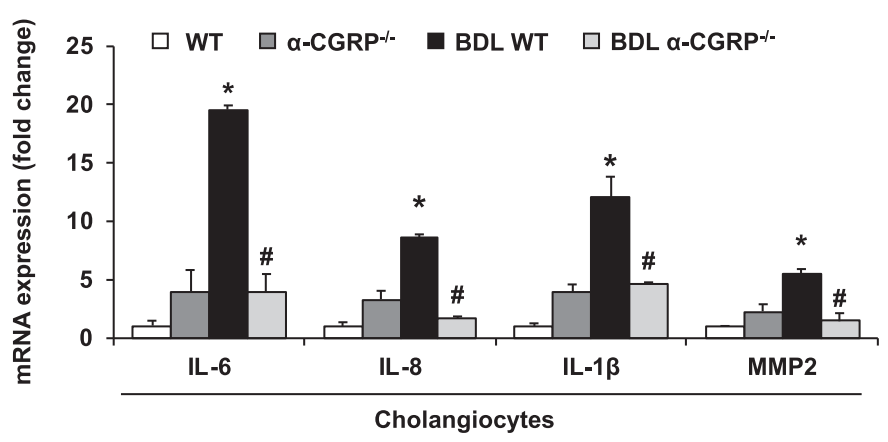

B
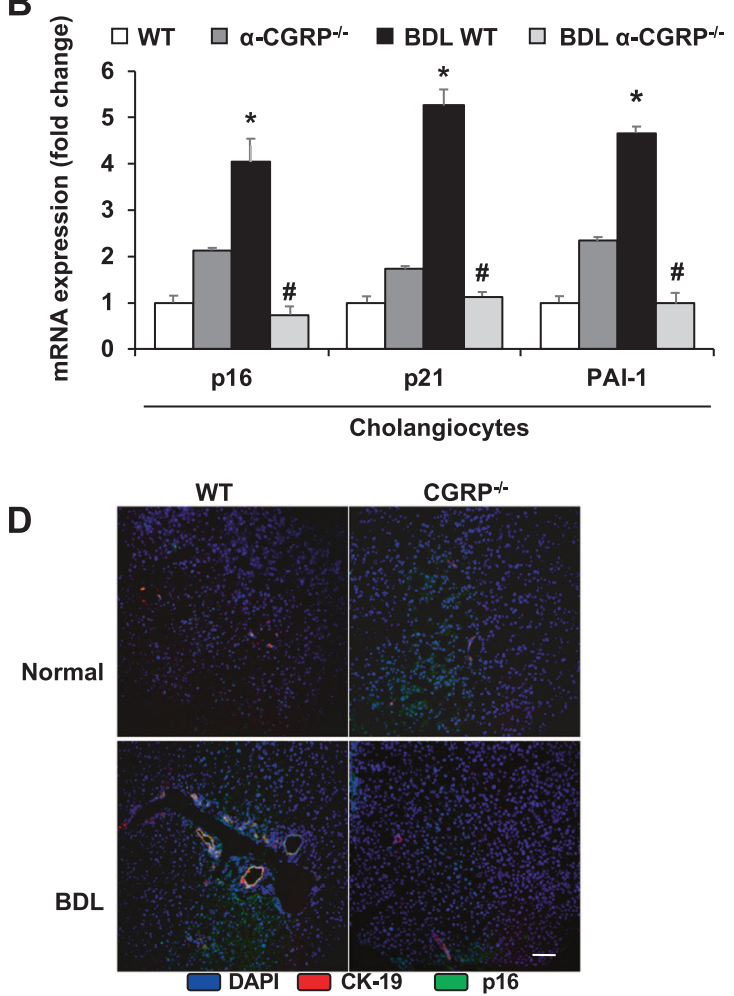

Fig. 5 Lack of $\alpha$-CGRP decreases fibrosis and senescence marker expression in cholangiocytes from BDL mice. A The mRNA expression of $\alpha$-SMA, Col1 $\alpha 1$, and Fn1 was decreased in cholangiocytes from BDL $\alpha-\mathrm{CGRP}^{-/}$mice compared to BDL WT mice $(n=4)$. B The mRNA expression of $\mathrm{p} 16, \mathrm{p} 21$, and PAI-1 was decreased in cholangiocytes from BDL $\alpha$-CGRP ${ }^{-/-}$mice compared to BDL WT mice $(n=4)$. C The mRNA expression of SASP markers such as IL-6, IL-8,

of HSCs to convert quiescent HSCs into activated HSCs as happens in the BDL model that activates proliferative, senescent and fibrogenic events in cholangiocytes.

\section{Effect of a-CGRP and CGRP $_{8-37}$ on the expression of fibrosis and senescence genes in IMCLs and HHSCs}

We observed that receptor activity-modifying protein 1 (RAMP-1) is expressed by both IMCLs and HHSCs (Fig. 7A). To determine the direct effect of $\alpha$-CGRP and CGRP $_{8-37}$ on cholangiocytes and HHSCs in vitro, we treated IMCLs and HHSCs with $\alpha$-CGRP $\left(10^{-9} \mathrm{M}\right)$ with or without the CGRP receptor antagonist $\left(\mathrm{CGRP}_{8-37}, 1 \mu \mathrm{M}\right)$ for $24 \mathrm{~h}$ before measuring fibrosis and senescence mRNA expression by $q$ PCR. Treatment of IMCLs and HHSCs with $\alpha$-CGRP $\left(10^{-9} \mathrm{M}\right.$ for $\left.24 \mathrm{~h}\right)$ increased the expression of $\alpha$ SMA and Col1 $\alpha 1$ in both cell types, increase that was partially reduced by treatment with $\mathrm{CGRP}_{8-37}$ (Fig. 7B, C). The profibrogenic effects of $\alpha$-CGRP on IMCLs and HHSCs were associated with increased senescence of
IL1- $\beta$, and MMP2 was decreased in cholangiocytes from BDL $\alpha-\mathrm{CGRP}^{-/-}$mice compared to BDL WT mice $(n=4)$. D Immunofluorescence demonstrated that p16 protein expression was decreased in cholangiocytes from BDL $\alpha-\mathrm{CGRP}^{-/-}$mice compared to BDL WT mice $(n=3$, Orig. magnification, $20 \times$, scale bar $=50 \mu \mathrm{m}) . * p<0.05$ vs. WT mice; ${ }^{\#} p<0.05$ vs. BDL WT mice

IMCLs but reduced senescence of HHSCs; the effects were prevented by incubation with $\mathrm{CGRP}_{8-37}$ (Fig. 7D).

\section{Effect of cholangiocyte supernatant on the expression of senescence and fibrosis markers in HHSCs}

When HHSCs were treated with cholangiocyte supernatant from BDL WT mice, there was decreased mRNA expression of the senescent markers, p16, p18, and p21, (Fig. 8D, F) and increased expression of the fibrotic genes, TGF- $\beta 1$, TIMP1, and Fn1, compared to HHSCs treated with cholangiocyte supernatant from WT mice (Fig. 8A-C). The extent of these increases was reduced when HHSCs were treated with cholangiocyte supernatant from BDL $\alpha-\mathrm{CGRP}^{-/-}$mice compared to HHSCs treated with cholangiocyte supernatant from BDL WT mice (Fig. 8A-F). The protein expression of Fn1 was decreased, whereas the expression of p16 was increased in HHSCs treated with cholangiocyte supernatant from BDL $\alpha-\mathrm{CGRP}^{-{ }^{--}}$mice 


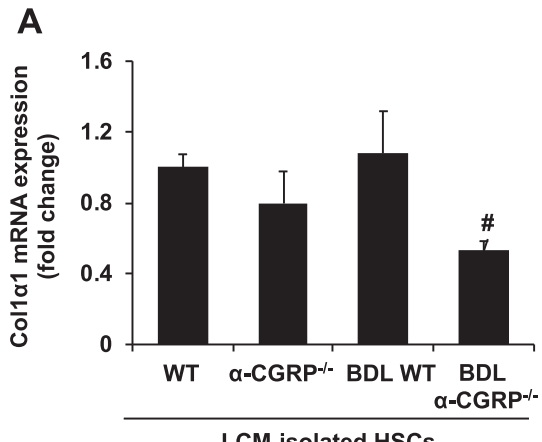

LCM-isolated HSCs
B

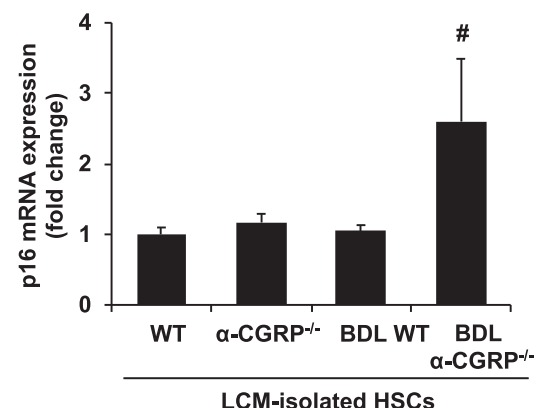

LCM-isolated HSCs

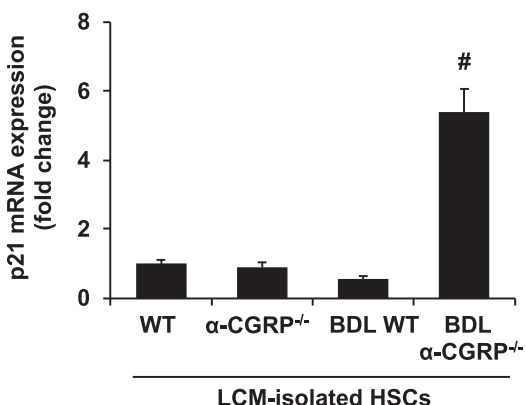

LCM-isolated HSCs
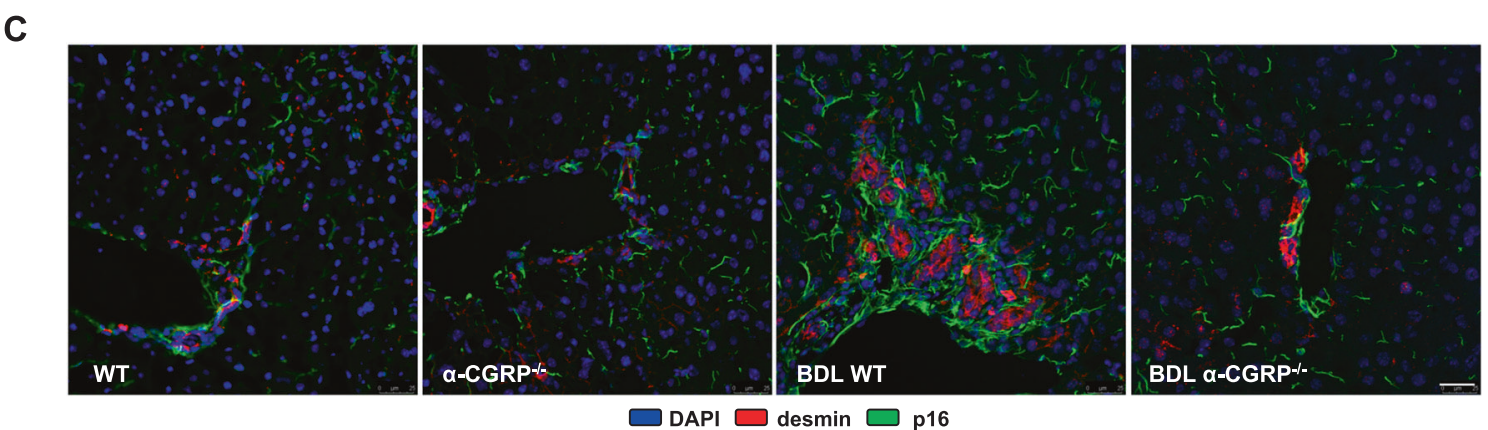

Fig. 6 Lack of $\alpha$-CGRP decreases fibrosis gene expression and increases senescence marker expression in HSCs from BDL mice. A The mRNA expression of Col $1 \alpha 1$ was decreased in HSCs isolated from BDL $\alpha-\mathrm{CGRP}^{-/-}$mice compared to BDL WT mice $(n=3)$. B The p16 and p21 mRNA expression was increased in $\mathrm{BDL} \alpha-\mathrm{CGRP}^{-/-}$mice

compared to BDL WT mice $(n=3)$. C Immunofluorescent staining showed that $\mathrm{p} 16$ protein expression was decreased in HSCs from BDL WT mice compared to BDL $\alpha-\mathrm{CGRP}^{-/-}$mice $(n=3$, Orig. magnification, $40 \times$, scale bar $=25 \mu \mathrm{m}) .{ }^{*} p<0.05$ vs. BDL WT mice

compared to HHSCs treated with cholangiocyte supernatant from BDL WT mice (Supplemental Fig. 3A, B).

\section{Discussion}

Liver fibrosis is a hallmark of acute and chronic liver diseases and is accompanied by a multicellular response with the activation of HSCs as a critical component $[27,28]$. Therefore, inhibition of the accumulation of activated HSCs by modulating either their activation and/ or proliferation, or by promoting HSC apoptosis and senescence, is an important potential target in patients with hepatic fibrosis. In this study, we demonstrated that there was increased hepatic expression of Calca (coding $\alpha$-CGRP) in BDL mice compared to normal WT mice. Furthermore, the expression of CGRP receptor components are elevated in $\alpha-\mathrm{CGRP}^{-/-}$mice as a compensatory response due to lacking of $\alpha$-CGRP. We also demonstrated an increase in CGRP serum levels and hepatic mRNA expression of CALCA and CGRP receptor components in human PSC samples compared to healthy controls. Knock-out of $\alpha$-CGRP reduced liver injury and fibrosis in BDL mice that was associated with enhanced cellular senescence in HSCs along with inhibition of MAPKs expression and decreased senescence of cholangiocytes. Furthermore, treatment with cholangiocyte supernatant from BDL $\alpha-\mathrm{CGRP}^{-/-}$mice decreased the activation and increased the senescence of cell lines of human HSCs compared to human HSCs stimulated with cholangiocyte supernatant from BDL WT mice.

$\alpha$-CGRP is widely distributed in a variety of tissues in both mice and humans [29] and plays a key role as a vasodilator in regulating vascular tone and blood flow under pathophysiological conditions [30]. In addition, $\alpha$-CGRP is now recognized to be a pleiotropic peptide that enhances heart [31] and skeletal muscle [32] contraction, regulates the growth of various cell types [15, 33] and suppresses gastric acid secretion [34]. Using $\alpha$-CGRP ${ }^{-/-}$mice, we have previously shown that sensory innervation of the liver and biliary expression of $\alpha$-CGRP regulates cholangiocyte proliferation during cholestasis, where there is damage of cholangiocytes and activation of HSCs resulting in the progression of liver fibrosis [15]. In the present study, we demonstrated that knockout of $\alpha$-CGRP alleviated liver injury and hepatic fibrosis in cholestatic mice. Consistent with our findings, other studies have demonstrated that $\alpha$-CGRP contributes to interstitial fibrogenesis in obstructive nephropathy [17, 35]. To clarify the mechanisms underlying the reduction of liver fibrosis due to knockout of $\alpha$-CGRP in BDL mice, we focused on the changes of cellular senescence in HSCs and cholangiocytes since 
A
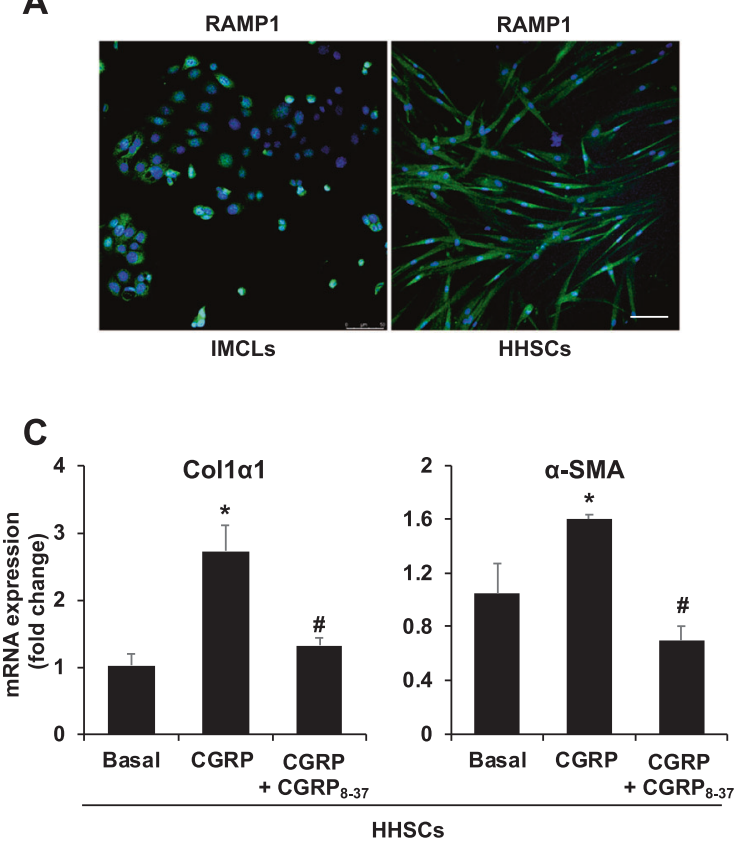

Fig. 7 Effect of $\alpha$-CGRP on the expression of fibrosis and senescence genes in cultured HHSCs and IMCLs. A Representative immunofluorescence picture of receptor activity-modifying protein 1 (RAMP$1)$ in HHSCs and IMCLs were shown $(n=4$, Orig., magnification. $40 \times$; scale bar $=50 \mu \mathrm{m}) . \mathbf{B}, \mathbf{C} \alpha$-CGRP stimulated the expression of

senescence of these cells have been shown to be involved in the development or regression of hepatic fibrosis [10, 12]. The differential expression of senescence markers between cholangiocytes and HSCs has been shown in several studies. For example, increased biliary senescence has been found in PSC and PBC patients [8, 11]. Also, increased senescence of activated HSCs can limit liver fibrosis induced by $\mathrm{CCl}_{4}$ treatment [6]. In this study, we demonstrated that lack of $\alpha$-CGRP increased senescence of HSCs and decreased biliary senescence, which likely explain why liver fibrosis is reduced in $\mathrm{BDL} \alpha-\mathrm{CGRP}^{-/-}$mice compared to BDL WT mice. Supporting our findings, another study has shown that increasing senescence of HSCs inhibits liver fibrosis after the treatment with the antiproliferative and apoptotic agent, dioscin [7]. Along with this notion, increased biliary proliferation has been suggested to trigger liver fibrosis in various chronic liver diseases [24, 36]. Damaged or proliferative cholangiocytes can express a number of profibrogenic and chemotactic proteins attracting both inflammatory cells and HSCs [37]. Cholangiocyte senescence and SASPs (i.e., senescence-associated secretory phenotypes) represent an important pathogenic mechanism in human cholestatic liver disorders including PSC [11]. On the basis of these previous studies, we propose that decreased cholangiocyte senescence and enhanced HSCs senescence after depletion of $\alpha$-CGRP both contribute to the reduction of liver fibrosis during cholestasis.

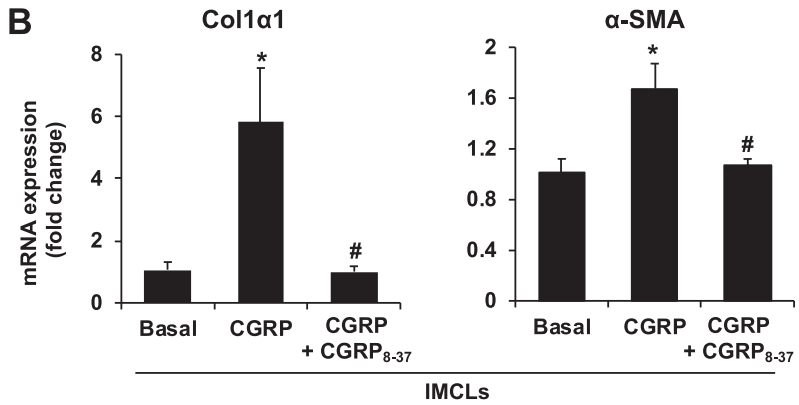

D Basal $\square$ CGRP $\square \mathrm{CGRP}+\mathrm{CGRP}_{8-37}$
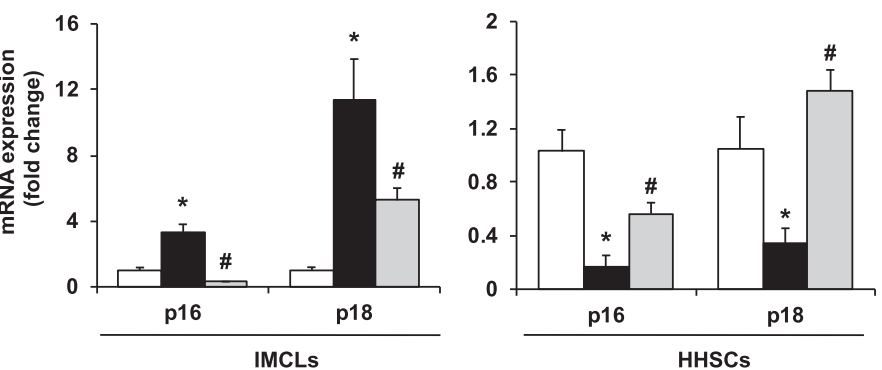

$\alpha$-SMA and Col1 $\alpha 1$ in both B IMCLs and C HHSCs, which was prevented by $\operatorname{CGRP}_{8-37}(n=4)$. D The expression of p16 and p18 was decreased in HHSCs while increased in ICMLs simulated by $\alpha$-CGRP; these effects were partly reversed by incubation with $\mathrm{CGRP}_{8-37}(n=$ 4). ${ }^{*} p<0.05$ vs. Basal; ${ }^{\#} p<0.05$ vs. $\alpha$-CGRP-treated group

We also propose that the sensory nerves containing substance $\mathrm{P}$ works as a profibrotic/compensatory factor by regulating the balance between the senescence of cholangiocytes and HSCs.

In our study, we also observed that cholangiocyte supernatant from BDL $\alpha-\mathrm{CGRP}^{-/}$mice decreased the activation of HHSCs through increasing senescence of these cells compared to HHSCs treated with cholangiocyte supernatant from BDL WT mice, which suggests that decreased chemotactic factors secreted by cholangiocytes (due to knockout of $\alpha$-CGRP) may reduce the activation and enhanced the senescence of HSCs during cholestasis. This study provides the information about the contribution of $\alpha$-CGRP on liver fibrosis and cellular senescence during cholestatic liver injury. However, other studies have demonstrated that $\alpha$-CGRP plays an anti-fibrotic role in pulmonary fibrosis [38] and in liver fibrosis due to hepatitis induced by repeated administration of concanavalin A [39]. This discrepant role of $\alpha$-CGRP-regulated in fibrosis may be due to mediators of different organs and different animal models of liver injury used. Thus, our study extends more understanding of $\alpha$-CGRP in liver fibrosis as well as in cellular senescence changes during cholestasis observed in our previous study [10].

In conclusion, this study provides evidence that $\alpha$-CGRP plays an important role in the regulation of biliary fibrosis and cellular senescence during cholestatic 

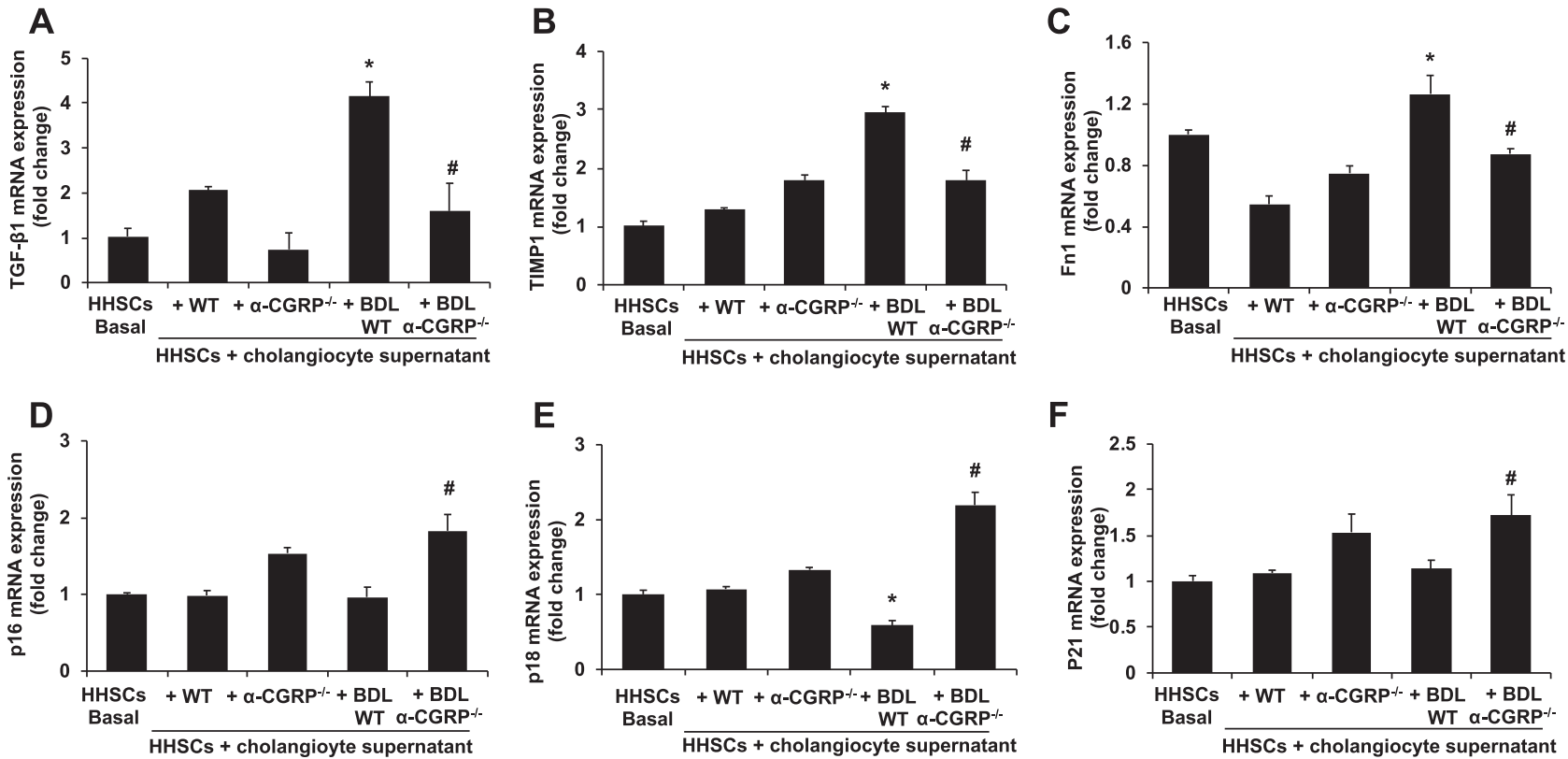

Fig. 8 Effect of stimulation with cholangiocyte supernatant on fibrosis and senescence gene expression in HHSCs. A-C The mRNA expression of A TGF- $\beta 1$, B TIMP1, and $\mathbf{C}$ Fn 1 increased in HHSCs stimulated with cholangiocyte supernatant from BDL WT mice compared to HHSCs stimulated with cholangiocyte supernatant from WT mice but decreased in HHSCs treated with cholangiocyte from BDL $\alpha$ $\mathrm{CGRP}^{-/-}$mice compared to HHSCs stimulated with cholangiocyte

liver injury. Biliary liver damage increases the expression of $\alpha$-CGRP, enhancing activation and decreasing senescence of HSCs, which results in liver fibrosis. Knockout $\alpha$-CGRP reverses liver fibrosis following cholestatic liver injury through reduced senescence of cholangiocytes and increased senescence of HSCs. Modulation of $\alpha$-CGRP/ CGRP receptor and its associated downstream signaling pathways may be an important therapeutic strategy for the management of biliary senescence and liver fibrosis in cholangiopathies.

\section{Disclaimer}

The content is the responsibility of the author(s) alone and does not necessarily reflect the views or policies of the Department of Veterans Affairs or the United States Government.

Acknowledgements This work was supported by the Dr. Nicholas C. Hightower Centennial Chair of Gastroenterology from Baylor Scott \& White, a VA Research Career Scientist Award and a VA Merit award to Dr. Alpini (5I01BX000574), a VA Merit Award (1I01BX001724) to Dr. Meng, and the NIH grants DK058411, DK076898, DK107310, DK110035, DK062975, AA025997, and AA025157 to Drs. Alpini, Meng, and Glaser. Portions of this work were supported by (i) a VA Merit Award (1I01BX003031) from the United States Department of Veteran's affairs, Biomedical Laboratory Research and Development Service and an R01 from NIH NIDDK (DK108959) to Dr. Francis (ii)

supernatant from BDL WT mice $(n=4)$. D-F The mRNA expression of D p16, E p18, and $\mathbf{F}$ p21 was increased in HHSCs treated with cholangiocyte supernatant from BDL $\alpha-\mathrm{CGRP}^{-/-}$mice compared to HHSCs stimulated with BDL cholangiocyte supernatant $(n=4) .{ }^{*} p<$ 0.05 vs. HHSCs treated with cholangiocyte supernatant from WT mice; ${ }^{*} p<0.05$ vs. HHSCs treated with cholangiocyte supernatant from BDL WT mice

and a Nature Science foundation of China (No.81873563) to Dr. Wan. This material is the result of work supported with resources and the use of facilities at the Central Texas Veterans Health Care System, Temple, Texas. This study was also funded by PSC Partners Seeking a Cure.

\section{Compliance with ethical standards}

Conflict of interest The authors declare that they have no conflict of interest.

Publisher's note: Springer Nature remains neutral with regard to jurisdictional claims in published maps and institutional affiliations.

\section{References}

1. Mogler C, Wieland M, König C, Hu J, Runge A, Korn C, et al. Hepatic stellate cell-expressed endosialin balances fibrogenesis and hepatocyte proliferation during liver damage. EMBO Mol Med. 2015;7:332-8.

2. Friedman SL. Mechanisms of hepatic fibrogenesis. Gastroenterology. 2008;134:1655-69.

3. Rahimi RS, Rockey DC. Complications of cirrhosis. Curr Opin Gastroenterol. 2012;28:223-9.

4. Poynard T, Lebray P, Ingiliz P, Varaut A, Varsat B, Ngo Y, et al. Prevalence of liver fibrosis and risk factors in a general population using non-invasive biomarkers (FibroTest). BMC Gastroenterol. 2010;10:40.

5. Hernandez-Gea V, Friedman SL. Pathogenesis of liver fibrosis. Annu Rev Pathol. 2011;6:425-56.

6. Krizhanovsky V, Yon M, Dickins RA, Hearn S, Simon J, Miething C, et al. Senescence of activated stellate cells limits liver fibrosis. Cell. 2008;134:657-67. 
7. Zhang X, Han X, Yin L, Xu L, Qi Y, Xu Y, et al. Potent effects of dioscin against liver fibrosis. Sci Rep. 2015;5:9713.

8. Tabibian JH, Trussoni CE, O’Hara SP, Splinter PL, Heimbach JK, LaRusso NF. Characterization of cultured cholangiocytes isolated from livers of patients with primary sclerosing cholangitis. Lab Invest. 2014;94:1126-33.

9. Baghdasaryan A, Claudel T, Gumhold J, Silbert D, Adorini L, Roda A, et al. Dual farnesoid X receptor/TGR5 agonist INT-767 reduces liver injury in the $\mathrm{Mdr}^{-{ }^{--}}\left(\mathrm{Abcb}^{-{ }^{--}}\right)$mouse cholangiopathy model by promoting biliary $\mathrm{HCO}(-)(3)$ output. Hepatology. 2011;54:1303-12.

10. Wan Y, Meng F, Wu N, Zhou T, Venter J, Francis H, et al. Substance $\mathrm{P}$ increases liver fibrosis by differential changes in senescence of cholangiocytes and hepatic stellate cells. Hepatology. 2017;66:528-41.

11. Tabibian JH, O'Hara SP, Splinter PL, Trussoni CE, LaRusso NF. Cholangiocyte senescence by way of N-ras activation is a characteristic of primary sclerosing cholangitis. Hepatology. 2014;59: 2263-75.

12. Meng L, Quezada M, Levine P, Han Y, McDaniel K, Zhou T, et al. Functional role of cellular senescence in biliary injury. Am J Pathol. 2015;185:602-9.

13. Holzer P. Local effector functions of capsaicin-sensitive sensory nerve endings: involvement of tachykinins, calcitonin gene-related peptide and other neuropeptides. Neuroscience. 1988;24:739-68.

14. Barry CM, Kestell G, Gillan M, Haberberger RV, Gibbins IL. Sensory nerve fibers containing calcitonin gene-related peptide in gastrocnemius, latissimus dorsi and erector spinae muscles and thoracolumbar fascia in mice. Neuroscience. 2015;291:106-17.

15. Glaser SS, Ueno Y, DeMorrow S, Chiasson VL, Katki KA, Venter J, et al. Knockout of alpha-calcitonin gene-related peptide reduces cholangiocyte proliferation in bile duct ligated mice. Lab Invest. 2007;87:914-26.

16. Henriksen JH, Schifter S, Møller S, Bendtsen F. Increased circulating calcitonin in cirrhosis. Relation to severity of disease and calcitonin gene-related peptide. Metabolism. 2000;49:47-52.

17. Yoon SP, Kim J Exogenous CGRP upregulates profibrogenic growth factors through $\mathrm{PKC} / \mathrm{JNK}$ signaling pathway in kidney proximal tubular cells. Cell Biol Toxicol 2017;4:251-62.

18. Glaser S, Benedetti A, Marucci L, Alvaro D, Baiocchi L, Kanno $\mathrm{N}$, et al. Gastrin inhibits cholangiocyte growth in bile duct-ligated rats by interaction with cholecystokinin-B/Gastrin receptors via $\mathrm{D}$ myo-inositol 1,4,5-triphosphate-, $\mathrm{Ca}\left({ }^{2+}\right)$-, and protein kinase $\mathrm{C}$ alpha-dependent mechanisms. Hepatology. 2000;32:17-25.

19. Puche JE, Lee YA, Jiao J, Aloman C, Fiel MI, et al. A novel murine model to deplete hepatic stellate cells uncovers their role in amplifying liver damage in mice. Hepatology. 2013;57:339-50.

20. Schmitt-Gräff A, Krüger S, Bochard F, Gabbiani G, Denk H. Modulation of alpha smooth muscle actin and desmin expression in perisinusoidal cells of normal and diseased human livers. Am J Pathol. 1991;138:1233-42.

21. Zhang L, Hoff AO, Wimalawansa SJ, Cote GJ, Gagel RF, Westlund KN. Arthritic calcitonin/alpha calcitonin gene-related peptide knockout mice have reduced nociceptive hypersensitivity. Pain. 2001;89:265-73.

22. Glaser S, Gaudio E, Renzi A, Mancinelli R, Ueno Y, Venter J. Knockout of the neurokinin-1 receptor reduces cholangiocyte proliferation in bile duct-ligated mice. Am J Physiol Gastrointest Liver Physiol. 2011;301:G297-305.

23. Glaser S, Meng F, Han Y, Onori P, Chow BK, Francis H, et al. Secretin stimulates biliary cell proliferation by regulating expression of microRNA $125 \mathrm{~b}$ and microRNA let7a in mice. Gastroenterology. 2014;146:1795-808 e1712.

24. Wu N, Meng F, Invernizzi P, Bernuzzi F, Venter J, Standeford H, et al. The secretin/secretin receptor axis modulates liver fibrosis through changes in transforming growth factor-betal biliary secretion in mice. Hepatology. 2016;64:865-79.

25. Ballardini G, Fallani M, Biagini G, Bianchi FB, Pisi E. Desmin and actin in the identification of Ito cells and in monitoring their evolution to myofibroblasts in experimental liver fibrosis. Virchows Arch B Cell Pathol Incl Mol Pathol. 1988;56:45-49.

26. Ehrlich L, O'Brien A, Hall C, White T, Chen L, Wu N, et al. alpha7-nAChR Knockout Mice Decreases Biliary Hyperplasia and Liver Fibrosis in Cholestatic Bile Duct-Ligated Mice. Gene Expr. 2018;18:197-207.

27. Bansal R, Prakash J, De Ruiter M, Poelstra K. Interferon gamma peptidomimetic targeted to hepatic stellate cells ameliorates acute and chronic liver fibrosis in vivo. J Control Release. 2014;179:18-24.

28. Mitra A, Satelli A, Yan J, Xueqing X, Gagea M, Hunter CA, et al. IL-30 (IL27p28) attenuates liver fibrosis through inducing NKG2D-rae1 interaction between NKT and activated hepatic stellate cells in mice. Hepatology. 2014;60:2027-39.

29. Li WW, Guo TZ, Shi X, Birklein F, Schlereth T, Kingery WS, et al. Neuropeptide regulation of adaptive immunity in the tibia fracture model of complex regional pain syndrome. J Neuroinflammation. 2018;15:105.

30. Sheykhzade M, Abdolalizadeh B, Koole C, Pickering DS, Dreisig $\mathrm{K}$, Johansson SE, et al. Vascular and molecular pharmacology of the metabolically stable CGRP analogue, SAX. Eur J Pharmacol. 2018;829:85-92.

31. Franco-Cereceda A, Lundberg JM. Calcitonin gene-related peptide (CGRP) and capsaicin-induced stimulation of heart contractile rate and force. Naunyn Schmiedebergs Arch Pharmacol. 1985;331:146-51.

32. Andersen SL, Clausen T. Calcitonin gene-related peptide stimulates active $\mathrm{Na}(+)-\mathrm{K}+$ transport in rat soleus muscle. Am J Physiol Cell Physiol. 1993;264:C419-429. (2 Pt 1)

33. Li Y, Fiscus RR, Wu J, Yang L, Wang X. The antiproliferative effects of calcitonin gene-related peptide in different passages of cultured vascular smooth muscle cells. Neuropeptides. 1997;31:503-9.

34. Tache Y. Inhibition of gastric acid secretion and ulcers by calcitonin [correction of calciton] gene-related peptide. Ann N Y Acad Sci. 1992;657:240-7.

35. Kim J, Padanilam BJ. Renal nerves drive interstitial fibrogenesis in obstructive nephropathy. J Am Soc Nephrol. 2013;24:229-42.

36. Sasaki M, Ikeda H, Yamaguchi J, Miyakoshi M, Sato Y, Nakanuma Y. Bile ductular cells undergoing cellular senescence increase in chronic liver diseases along with fibrous progression. Am J Clin Pathol. 2010;133:212-23.

37. Beuers U. Crosstalk of liver, bile ducts and the gut. Clin Rev Allergy Immunol. 2009;36:1-3.

38. Li XW, Li XH, Du J, Li D, Li YJ, Hu CP. Calcitonin gene-related peptide down-regulates bleomycin-induced pulmonary fibrosis. Can J Physiol Pharmacol. 2016;94:1315-24.

39. Kamiyoshi A, Sakurai T, Ichikawa-Shindo Y, Iinuma N, Kawate $\mathrm{H}$, Yoshizawa T, et al. Endogenous alpha-calcitonin gene-related peptide mitigates liver fibrosis in chronic hepatitis induced by repeated administration of concanavalin A. Liver Int. 2009;29:642-9. 


\section{Affiliations}

Ying Wan ${ }^{1} \cdot$ Ludovica Ceci ${ }^{2} \cdot \mathrm{Nan}_{\mathrm{Wu}^{2}} \cdot{\text { Tianhao } \mathrm{Zhou}^{2} \cdot \text { Lixian } \mathrm{Chen}^{2} \cdot \text { Julie Venter }^{2} \cdot \text { Heather Francis }}^{2,3,4}$. Francesca Bernuzzi $i^{5}$ Pietro Invernizzi $\mathbb{1}^{5} \cdot$ Konstantina Kyritsi $^{2} \cdot$ Paul Baker $^{2}$ - Qiaobing Huang ${ }^{6}$. Chaodong $\mathrm{Wu}^{7}$. Amelia Sybenga $^{8} \cdot$ Gianfranco Alpini ${ }^{2,3,4} \cdot$ Fanyin Meng ${ }^{2,3,4,9} \cdot$ Shannon Glaser ${ }^{2,4}$

1 Department of Pathophysiology, Southwest Medical University, Luzhou 646000, China

2 Department of Medical Physiology, Texas A\&M University Health Science Center, Temple, TX, USA

3 Research, Central Texas Veterans Health Care System, Temple, TX, USA

4 Digestive Disease Research Center, Baylor Scott \& White Health, Temple, TX, USA

5 Humanitas Clinical and Research Center, Rozzano (MI), Italy
6 Department of Pathophysiology, Key Lab for Shock and Microcirculation Research of Guangdong Province, Southern Medical University, Guangzhou, China

7 Department of Nutrition and Food Science, Texas A\&M University, College Station, TX 77843, USA

8 Department of Pathology, Microbiology, and Immunology, Vanderbilt University School of Medicine, Nashville, TN, USA

9 Research Foundation, Baylor Scott \& White Health, Temple, TX, USA 